\title{
Exact Solution for a Free Vibration of Thermoelas- tic Hollow Cylinder Under GNIII Model
}

\author{
Ibrahim A. Abbas \\ Department of Mathematics, Faculty of Science and Arts - Khulais, University Of Jeddah, Saudi Arabia \\ Nonlinear Analysis and Applied Mathematics Research Group (NAAM), Department of Mathematics, King Abdu- \\ laziz University, Jeddah, Saudi Arabia \\ Department of Mathematics, Faculty of Science, Sohag University, Sohag, Egypt.
}

(Received 12 April 2014; accepted 17 February 2015)

The exact analytic solutions are obtained with the use of the eigenvalue approach for a free vibration problem of a thermoelastic hollow cylinder in the context of Green and Naghdi theory (GNIII). The dispersion relations for the existence of various types of possible modes of vibrations in the considered hollow cylinder are derived in a compact form and the validation of the roots for the dispersion relation is presented. To illustrate the analytic results, the numerical solution of various relations and equations has been carried out to compute the frequency, thermoelastic damping and frequency shift of vibrations in a hollow cylinder of copper material with MATHEMATICA and MATLAB software.

\section{INTRODUCTION}

In the literature concerning thermal effects in continuum mechanics, several parabolic and hyperbolic theories for describing the heat conduction were developed. These hyperbolic theories were also called theories of second sound and there the flow of heat was modelled with finite propagation speed, which contrasts with the classical model based on the Fouriers law leading to infinite propagation speed of heat signals as in. ${ }^{1-9}$ Green and Naghdi ${ }^{10,11}$ proposed GNII and GNIII, which is a generalized thermoelasticity theory based on entropy equality rather than the usual entropy inequality. An important feature of this theory, which was not present in other thermoelasticity theories, was that it does not accommodate the dissipation of thermal energy. GN theory seems to be idealistic from a physical point of view. The genesis lies in the fact that the thermoelastic model of the GN theory was an idealized material model. During the last years, different problems were considered by using Green and Naghdi theories, as in Abd El-Latief et al., ${ }^{12}$ Youssef, ${ }^{13}$ Mukhopadhyay et al., ${ }^{14}$ Sharma et al., ${ }^{15}$ Prasad et al., ${ }^{16}$ Othman et al., ${ }^{17}$ Abbas, ${ }^{18}$ and Abbas et al. ${ }^{19}$ A survey article of representative theories in the range of generalized thermoelasticity is given by Hetnarski and Ignaczak. $^{20}$

The vibrations in thermoelastic materials have many applications in various fields of science and technology, namely aerospace, atomic physics, thermal power plants, and chemical pipes. The cylinders were frequently used as structural components and their vibrations were obviously important for practical design. Abbas studied the natural frequencies of a poroelastic hollow cylinder. ${ }^{21}$ Abd-alla and Abbas investigated the magnetoelastic longitudinal wave propagation in a transversely isotropic circular cylinder. ${ }^{22}$ Mykityuk studied the thermoelastic vibrations of a thick-walled cylinder of time-varying thickness. ${ }^{23}$ Zhitnyaya analyzed an uncoupled problem of the thermoelastic vibrations of a cylinder. ${ }^{24}$ Marin and Lupu studied the harmonic vibrations in thermoelasticity of micropolar bodies. ${ }^{25}$ Erbay et al. investigated thermally induced vibrations in a generalized thermoelastic solid with a cavity. ${ }^{26}$ Sharma et al. solved the vibration analysis of a transversely isotropic hollow cylinder by using the matrix Frobenius method. ${ }^{27}$ Nayfeh and Younis presented a model for thermoelastic damping in microplates. ${ }^{28,29}$ Rezazadeh et al. studied the thermoelastic damping in a micro-beam resonator using modified couple stress theory. ${ }^{30}$

The present article is devoted to study the frequency, frequency shifts and damping due to thermal variations in homogenous isotropic hollow cylinder, in the context of Green and Naghdi of type III model of non-classical (generalized) thermoelasticity.

\section{BASIC EQUATION AND FORMULATION OF THE PROBLEM}

Following Green and Naghdi, the basic equations of the thermoelasticity theory for homogeneous isotropic material in the absence of body forces and heat sources were considered as the equations of motion ${ }^{10,11}$ :

$$
\sigma_{i j, j}=\rho \frac{\partial^{2} u_{i}}{\partial t^{2}}
$$

where $\rho$ was the density of the medium, $t$ was the time, $\sigma_{i j}$ were the components of stress tensor, and $u_{i}$ were the components of displacement vector. The equation of heat conduction is:

$$
\left(K_{i j}^{*} T_{, j}+K_{i j} \dot{T}_{, j}\right)=\frac{\partial^{2}}{\partial t^{2}}\left(\rho c_{e}+\gamma T_{0} e\right) ;
$$

where $T$ is the temperature, $c_{e}$ was the specific heat at constant strain, $K_{i j}$ was the thermal conductivity, $K_{i j}^{*}$ was the material constant characteristic of the theory, $T_{0}$ was the reference temperature; $\gamma=(3 \lambda+2 \mu) \alpha_{t}$, $\alpha_{t}$ was the coefficient of linear thermal expansion. The constitutive equations were given by:

$$
\sigma_{i j}=2 \mu e_{i j}+\left[\lambda e-\gamma\left(T-T_{0}\right)\right] \delta_{i j} ;
$$

with $e=e_{i i}, i, j=r, \theta, z$, where $\lambda, \mu$ were the Lame's constants and $\delta_{i j}$ was the Kronecker symbol. 
Let us consider an elastic hollow cylinder of an isotropic homogeneous medium whose state could be expressed in terms of the space variable $r$ and the time variable $t$. In a cylindrical coordinate system $(r, \theta, z)$, for the axially symmetric problem $u_{r}=u_{r}(r, z, t), u_{\theta}=0, u_{z}=u_{z}(r, z, t)$. Furthermore, if only the axisymmetric plane strain problem was considered, we had $u_{r}=u(r, t)$ and $u_{\theta}=u_{z}=0$. Thus, the straindisplacement relations are

$$
e_{r r}=\frac{\partial u}{\partial r}, \quad e_{\theta \theta}=\frac{u}{r}, \quad e_{z z}=e_{r z}=e_{r \theta}=e_{\theta z}=0 .
$$

The stress-strain relations are

$$
\begin{aligned}
\sigma_{r r} & =2 \mu \frac{\partial u}{\partial r}+\lambda\left(\frac{\partial u}{\partial r}+\frac{u}{r}\right)-\gamma\left(T-T_{0}\right) ; \\
\sigma_{\theta \theta} & =2 \mu \frac{u}{r}+\lambda\left(\frac{\partial u}{\partial r}+\frac{u}{r}\right)-\gamma\left(T-T_{0}\right) .
\end{aligned}
$$

It was assumed that there were no body forces and heat sources in the medium, the equation of motion and energy equation hadthe form:

$$
\begin{gathered}
\frac{\partial \sigma_{r r}}{\partial r}+\frac{\sigma_{r r}-\sigma_{\theta \theta}}{r}=\rho \frac{\partial^{2} u}{\partial t^{2}} \\
K^{*} \frac{1}{r} \frac{\partial}{\partial r}\left(r \frac{\partial T}{\partial r}\right)+K \frac{1}{r} \frac{\partial}{\partial r}\left(r \frac{\partial^{2} T}{\partial t \partial r}\right)= \\
\frac{\partial^{2}}{\partial t^{2}}\left(\rho c_{e} T+\gamma T_{0}\left(\frac{\partial u}{\partial r}+\frac{u}{r}\right)\right) .
\end{gathered}
$$

It was convenient to change the preceding equations into the dimensionless forms. To do this, the dimensionless parameters were introduced as

$$
\begin{gathered}
\left(r^{\prime}, u^{\prime}\right)=\frac{(r, u)}{c \chi}, \quad t^{\prime}=\frac{t}{\chi}, \quad \omega^{\prime}=\omega \chi, \\
\left(\sigma_{r r}^{\prime}, \sigma_{\theta \theta}^{\prime}\right)=\frac{1}{\lambda+2 \mu}\left(\sigma_{r r}, \sigma_{\theta \theta}\right), \quad T^{\prime}=\frac{T-T_{0}}{T_{0}},
\end{gathered}
$$

where, $c^{2}=\frac{\lambda+2 \mu}{\rho}, \chi=\frac{K}{\rho c_{e} c^{2}}$. From Eq. (9) into Eqs. (5) to (8), one may obtain (here dashes are ignored for convenience):

$$
\begin{gathered}
\frac{\partial^{2} u}{\partial r^{2}}+\frac{1}{r} \frac{\partial u}{\partial r}-\frac{u}{r^{2}}-a_{2} \frac{\partial T}{\partial r}=\frac{\partial^{2} u}{\partial t^{2}} \\
\left(\varepsilon_{1}+\frac{\partial}{\partial t}\right)\left(\frac{\partial^{2} T}{\partial r^{2}}+\frac{1}{r} \frac{\partial T}{\partial r}\right)=\frac{\partial^{2}}{\partial t^{2}}\left(T+\varepsilon_{2}\left(\frac{\partial u}{\partial r}+\frac{u}{r}\right)\right)
\end{gathered}
$$$$
\sigma_{r r}=\frac{\partial u}{\partial r}+a_{1} \frac{u}{r}-a_{2} T
$$$$
\sigma_{\theta \theta}=a_{1} \frac{\partial u}{\partial r}+\frac{u}{r}-a_{2} T
$$

where $a_{1}=\frac{\lambda}{\lambda+2 \mu}, a_{2}=\frac{\gamma T_{0}}{\lambda+2 \mu}, \varepsilon_{1}=\frac{K^{*}}{\rho c_{e} c^{2}}, \varepsilon_{2}=\frac{\gamma}{\rho c_{e}}$. The boundary conditions for stress free and isothermal surfaces of the cylinder may be expressed as:

$$
\sigma_{r r}(a, t)=\sigma_{r r}(b, t)=0 ; \quad T(a, t)=T(b, t)=0 ;
$$

where $a$ and $b$ are the inner and outer radii of the cylinder respectively.

\section{THE EXACT SOLUTION OF THE MODEL}

We considered cylindrical time-harmonic vibrations so that:

$$
u(r, t)=\bar{u}(r) e^{i \omega t}, \quad T(r, t)=\bar{T} e^{i \omega t} ;
$$

where $\omega$ was the non-dimensional circular frequency of vibrations. By placing Eq. (15) into Eqs. (10) and (11), we get:

$$
\begin{gathered}
\frac{d^{2} \bar{u}}{d r^{2}}+\frac{1}{r} \frac{d \bar{u}}{d r}-\frac{\bar{u}}{r^{2}}=-\omega^{2} \bar{u}+a_{2} \frac{d \bar{T}}{d r} \\
\frac{d^{2} \bar{T}}{d r^{2}}+\frac{1}{r} \frac{d \bar{T}}{d r}=-\omega^{2}\left(\varepsilon_{3} \bar{T}+\varepsilon_{4}\left(\frac{d \bar{u}}{d r}+\frac{\bar{u}}{r}\right)\right)
\end{gathered}
$$

where $\varepsilon_{3}=\frac{1}{\varepsilon_{1}+i \omega}$ and $\varepsilon_{4}=\frac{\varepsilon_{2}}{\varepsilon_{1}+i \omega}$. By differentiating Eq. (17) with respect to $r$ and using Eq. (16) we got:

$$
\begin{aligned}
\frac{d^{2}}{d r^{2}}\left(\frac{d \bar{T}}{d r}\right)+ & \frac{1}{r} \frac{d}{d r}\left(\frac{d \bar{T}}{d r}\right)-\frac{1}{r^{2}}\left(\frac{d \bar{T}}{d r}\right)= \\
& -\omega^{2}\left(-\omega^{2} \varepsilon_{4} \bar{u}+\left(\varepsilon_{3}+\varepsilon_{4} a_{2}\right) \frac{d \bar{T}}{d r}\right) .
\end{aligned}
$$

Equations (16) and (18) could be written in a vector-matrix differential equation as follows:

$$
L \mathbf{V}=\mathbf{A V}
$$

where $L \equiv \frac{d^{2}}{d r^{2}}+\frac{1}{r} \frac{d}{d r}-\frac{1}{r^{2}}$ was the Bessel operator, $\mathbf{V}=$ $\left[\begin{array}{ll}\bar{u} & \frac{d \bar{T}}{d r}\end{array}\right]^{T}$ and $\mathbf{A}=\left[\begin{array}{ll}A_{11} & A_{12} \\ A_{21} & A_{22}\end{array}\right]$, with $A_{11}=-\omega^{2}, A_{12}=$ $a_{2}, A_{21}=\omega^{4} \varepsilon_{4}, A_{22}=-\omega^{2}\left(\varepsilon_{3}+a_{2} \varepsilon_{4}\right)$.

Let us now proceed to solve Eq. (19) by the eigenvalue approach proposed by Das at al., ${ }^{31}$ Abbas, ${ }^{32-34,36}$ and Youssef et al. ${ }^{35}$ The characteristic equation of the matrix $\mathbf{A}$ takes the form:

$$
A_{11} A_{22}-A_{12} A_{21}-\left(A_{22}+A_{11}\right) \lambda+\lambda^{2}=0 .
$$

The roots of the characteristic Eq. (20), which were also the eigenvalues of matrix $\mathbf{A}$, were of the form $\lambda=\lambda_{1}, \lambda=\lambda_{2}$. The eigenvector $\mathbf{X}=\left[\begin{array}{ll}x_{1} & x_{2}\end{array}\right]^{T}$, which corresponded to the eigenvalue $\lambda$, could be calculated as:

$$
x_{1}=A_{12}, \quad x_{2}=\lambda-A_{11} .
$$

From Eq. (20), we could easily calculate the eigenvector $\mathbf{X}_{j}$, which corresponded to the eigenvalue $\lambda_{j}, j=1,2$. For further reference, we shall use the following notations:

$$
\mathbf{X}_{1}=[\mathbf{X}]_{\lambda=\lambda_{1}}, \quad \mathbf{X}_{2}=[\mathbf{X}]_{\lambda=\lambda_{2}} .
$$

The solution of Eq. (20) could be written as follows:

$$
\begin{aligned}
\mathbf{V}=\mathbf{X}_{1}\left(A_{1} I_{1}\left(p_{1} r\right)+A_{2} K_{1}\left(p_{1} r\right)\right)+ \\
\mathbf{X}_{2}\left(A_{3} I_{1}\left(p_{2} r\right)+A_{4} K_{1}\left(p_{2} r\right)\right) ;
\end{aligned}
$$

where $p_{1}=\sqrt{\lambda_{1}}, p_{2}=\sqrt{\lambda_{2}}, I_{1}, K_{1}$ were the modified of Bessels functions and $A_{1}, A_{2}, A_{3}, A_{4}$ were arbitrary constants to be determined. Upon using Eq. (23), the displacement and temperature gradient were obtained as:

$$
\begin{aligned}
\bar{u}(r)=x_{1}^{1}\left(A_{1} I_{1}\left(p_{1} r\right)+A_{2} K_{1}\left(p_{1} r\right)\right)+ \\
x_{1}^{2}\left(A_{3} I_{1}\left(p_{2} r\right)+A_{4} K_{1}\left(p_{2} r\right)\right) ;
\end{aligned}
$$




$$
\begin{aligned}
& \frac{d \bar{T}}{d r}=x_{2}^{1}\left(A_{1} I_{1}\left(p_{1} r\right)+A_{2} K_{1}\left(p_{1} r\right)\right)+ \\
& x_{2}^{2}\left(A_{3} I_{1}\left(p_{2} r\right)+A_{4} K_{1}\left(p_{2} r\right)\right) ;
\end{aligned}
$$

where $x_{i}^{j}$ was the component number $i$ of the eigenvector number $j$. Thus, the exact solutions of field variables could be written for $r$ and $t$ as:

$$
\begin{aligned}
& u(r, t)=\left[x_{1}^{1}\left(A_{1} I_{1}\left(p_{1} r\right)+A_{2} K_{1}\left(p_{1} r\right)\right)+\right. \\
& \left.x_{1}^{2}\left(A_{3} I_{1}\left(p_{2} r\right)+A_{4} K_{1}\left(p_{2} r\right)\right)\right] e^{i \omega t} ; \\
& T(r, t)=\left[\frac{x_{2}^{1}}{p_{1}}\left(A_{1} I_{0}\left(p_{1} r\right)-A_{2} K_{0}\left(p_{1} r\right)\right)+\right. \\
& \left.\frac{x_{2}^{2}}{p_{2}}\left(A_{3} I_{0}\left(p_{2} r\right)-A_{4} K_{0}\left(p_{2} r\right)\right)\right] e^{i \omega t} ; \\
& \sigma_{\theta \theta}(r, t)= \\
& A_{1}\left[\frac{\left(p_{1}^{2} \xi x_{1}^{1}-\beta x_{2}^{1}\right)}{p_{1}} I_{0}\left(p_{1} r\right)-\frac{x_{1}^{1}(\xi-1)}{r} I_{1}\left(p_{1} r\right)\right]+ \\
& A_{2}\left[\frac{\left(\beta x_{2}^{1}-p_{1}^{2} \xi x_{1}^{1}\right)}{p_{1}} K_{0}\left(p_{1} r\right)-\frac{x_{1}^{1}(\xi-1)}{r} K_{1}\left(p_{1} r\right)\right]+ \\
& A_{3}\left[\frac{\left(p_{2}^{2} \xi x_{1}^{2}-\beta x_{2}^{2}\right)}{p_{2}} I_{0}\left(p_{2} r\right)-\frac{x_{1}^{2}(\xi-1)}{r} I_{1}\left(p_{2} r\right)\right]+ \\
& A_{4}\left[\frac{\left(\beta x_{2}^{2}-p_{2}^{2} \xi x_{1}^{2}\right)}{p_{2}} K_{0}\left(p_{2} r\right)-\frac{x_{1}^{2}(\xi-1)}{r} K_{1}\left(p_{2} r\right)\right] \text {. }
\end{aligned}
$$

\section{DISPERSION RELATIONS}

We assumed that the thermoelastic hollow cylinder was subjected to traction-free and isothermal boundary conditions, Eq. (15), at its surfaces $(r=a, b)$. By applying boundary conditions, which were Eqs. (15), (27), and (28), we obtain a system of four homogeneous linear algebraic equations in unknowns $A_{1}, A_{2}, A_{3}$, and $A_{4}$. This system would have a nontrivial solution if and only if the determinant of the coefficients $A_{1}, A_{2}, A_{3}$, and $A_{4}$ vanished and such a requirement of nontrivial solution lead to dispersion equations given by:

$$
\Delta=\operatorname{det}\left(L_{i j}\right)=0, \quad i, j=1,2,3,4 ;
$$

where,

$$
\begin{aligned}
& L_{11}=\frac{\left(p_{1}^{2} x_{1}^{1}-a_{2} x_{2}^{1}\right)}{p_{1}} I_{0}\left(p_{1} a\right)+\frac{x_{1}^{1}\left(a_{1}-1\right)}{a} I_{1}\left(p_{1} a\right) ; \\
& L_{12}=\frac{\left(a_{2} x_{2}^{1}-p_{1}^{2} x_{1}^{1}\right)}{p_{1}} K_{0}\left(p_{1} a\right)+\frac{x_{1}^{1}\left(a_{1}-1\right)}{a} K_{1}\left(p_{1} a\right) ;
\end{aligned}
$$

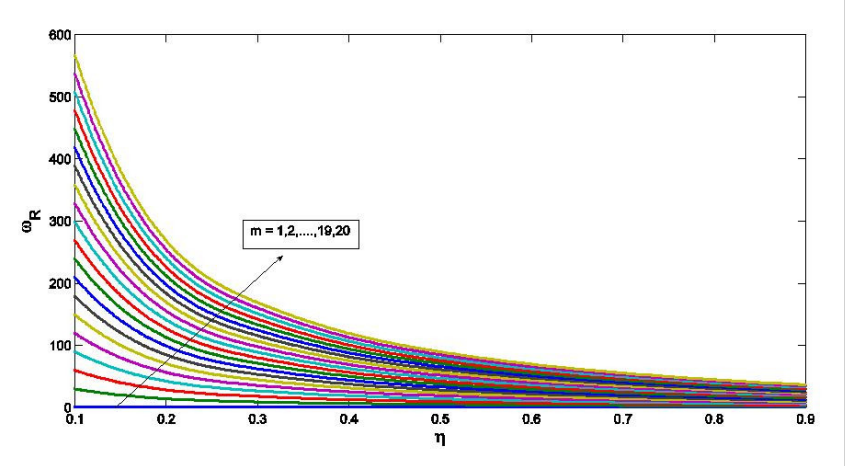

Figure 1. Non-dimensional frequency $\omega_{R}$ verses the length to mean radius ratio $\eta$.

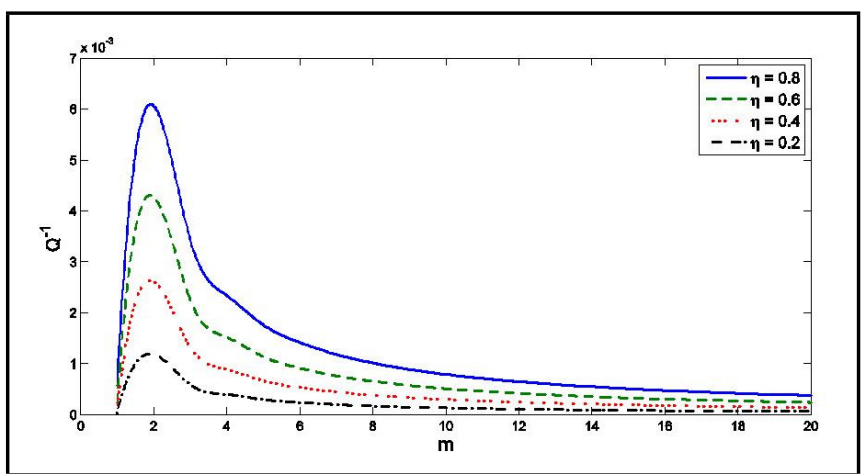

Figure 2. Thermoelastic damping $Q^{-1}$ versus $m$ for different values of $\eta$.

$$
\begin{gathered}
L_{13}=\frac{\left(p_{2}^{2} x_{1}^{2}-a_{2} x_{2}^{2}\right)}{p_{2}} I_{0}\left(p_{2} a\right)+\frac{x_{1}^{2}\left(a_{1}-1\right)}{a} I_{1}\left(p_{2} a\right) ; \\
L_{14}=\frac{\left(a_{2} x_{2}^{2}-p_{2}^{2} x_{1}^{2}\right)}{p_{2}} K_{0}\left(p_{2} a\right)+\frac{x_{1}^{2}\left(a_{1}-1\right)}{a} K_{1}\left(p_{2} a\right) ; \\
L_{21}=\frac{\left(p_{1}^{2} x_{1}^{1}-a_{2} x_{2}^{1}\right)}{p_{1}} I_{0}\left(p_{1} b\right)+\frac{x_{1}^{1}\left(a_{1}-1\right)}{b} I_{1}\left(p_{1} b\right) ; \\
L_{22}=\frac{\left(a_{2} x_{2}^{1}-p_{1}^{2} x_{1}^{1}\right)}{p_{1}} K_{0}\left(p_{1} b\right)+\frac{x_{1}^{1}\left(a_{1}-1\right)}{b} K_{1}\left(p_{1} b\right) ; \\
L_{23}=\frac{\left(p_{2}^{2} x_{1}^{2}-a_{2} x_{2}^{2}\right)}{p_{2}} I_{0}\left(p_{2} b\right)+\frac{x_{1}^{2}\left(a_{1}-1\right)}{b} I_{1}\left(p_{2} b\right) ; \\
L_{24}=\frac{\left(a_{2} x_{2}^{2}-p_{2}^{2} x_{1}^{2}\right)}{p_{2}} K_{0}\left(p_{2} b\right)+\frac{x_{1}^{2}\left(a_{1}-1\right)}{b} K_{1}\left(p_{2} b\right) ; \\
L_{31}=\frac{x_{2}^{1}}{p_{1}} I_{0}\left(p_{1} a\right), \quad L_{32}=-\frac{x_{2}^{1}}{p_{1}} K_{0}\left(p_{1} a\right) \\
L_{33}=\frac{x_{2}^{2}}{p_{2}} I_{0}\left(p_{2} a\right), \quad L_{34}=-\frac{x_{2}^{2}}{p_{2}} K_{0}\left(p_{2} a\right) \\
L_{41}=\frac{x_{2}^{1}}{p_{1}} I_{0}\left(p_{1} b\right), \quad L_{42}=-\frac{x_{1}^{2}}{p_{1}} K_{0}\left(p_{1} b\right) \\
L_{43}=\frac{x_{2}^{2}}{p_{2}} I_{0}\left(p_{2} b\right), \quad L_{44}=-\frac{x_{2}^{2}}{p_{2}} K_{0}\left(p_{2} b\right)
\end{gathered}
$$

\section{NUMERICAL RESULTS AND DISCUSSION}

The copper material had been chosen for the purposes of numerical evaluations in the space-time domain. From the material constants, we got the non-dimensional values of the problem as Abbas: ${ }^{37}$

$$
\mu=3.86 \times 10^{10}(\mathrm{~kg})(\mathrm{m})^{-1}(\mathrm{~s})^{-2}
$$


Table 1. The validation of the roots for the dispersion relation.

\begin{tabular}{|c|c|c|c|c|}
\hline \multirow{2}{*}{$\mathrm{m}$} & \multicolumn{2}{|c|}{$\eta=0.4$} & \multicolumn{2}{c|}{$\eta=0.8$} \\
\cline { 2 - 5 } & $\omega$ & $\Delta$ & $\omega$ & $\Delta$ \\
\hline 1 & $0.7062+0.00002 \mathrm{i}$ & $4.2 \times 10^{-16}-1.5 \times 10^{-17} \mathrm{i}$ & $0.5628+0.00016 \mathrm{i}$ & $9.9 \times 10^{-16}+1.1 \times 10^{-15} \mathrm{i}$ \\
2 & $6.3247+0.00825 \mathrm{i}$ & $-3.2 \times 10^{-16}-6.9 \times 10^{-16} \mathrm{i}$ & $2.4331+0.00738 \mathrm{i}$ & $-1.0 \times 10^{-16}+1.8 \times 10^{-17} \mathrm{i}$ \\
5 & $25.143+0.00839 \mathrm{i}$ & $5.1 \times 10^{-19}-2.4 \times 10^{-19} \mathrm{i}$ & $9.4425+0.00834 \mathrm{i}$ & $5.0 \times 10^{-16}-7.1 \times 10^{-17} \mathrm{i}$ \\
10 & $56.553+0.00839 \mathrm{i}$ & $-23 \times 10^{-18}-4.4 \times 10^{-19} \mathrm{i}$ & $21.213+0.00839 \mathrm{i}$ & $-2.0 \times 10^{-18}+7.1 \times 10^{-18} \mathrm{i}$ \\
15 & $87.967+0.0084 \mathrm{i}$ & $7.3 \times 10^{-19}+1.3 \times 10^{-18} \mathrm{i}$ & $32.991+0.00839 \mathrm{i}$ & $1.7 \times 10^{-17}+4.8 \times 10^{-18} \mathrm{i}$ \\
20 & $119.38+0.0084 \mathrm{i}$ & $-3.1 \times 10^{-19}-1.8 \times 10^{-18} \mathrm{i}$ & $44.771+0.00839 \mathrm{i}$ & $-2.7 \times 10^{-17}+1.5 \times 10^{-18} \mathrm{i}$ \\
\hline
\end{tabular}

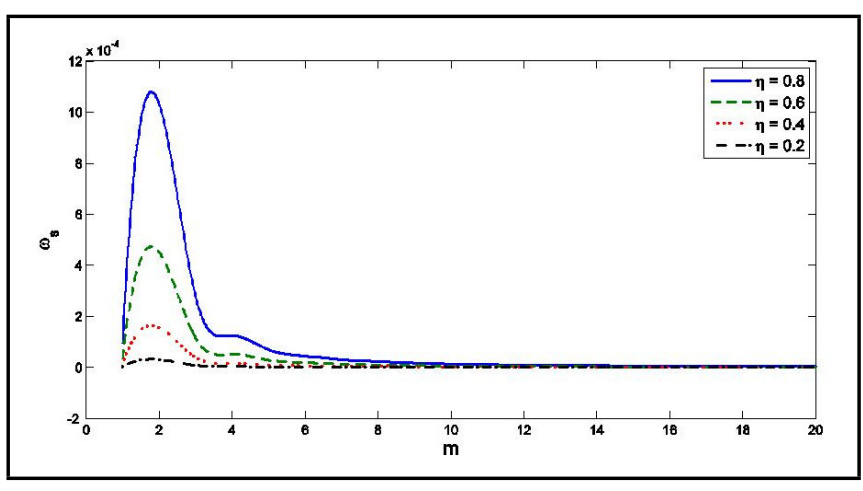

Figure 3. Frequency shift $\omega_{s}$ versus $m$ for different values of $\eta$

$$
\begin{aligned}
\lambda & =7.76 \times 10^{10}(\mathrm{~kg})(\mathrm{m})^{-1}(\mathrm{~s})^{-2} ; \\
c_{e} & =3.831 \times 10^{2}(\mathrm{~m})^{2}(\mathrm{~K})^{-1}(\mathrm{~s})^{-2} ; \\
K & =3.68 \times 10^{2}(\mathrm{~kg})(\mathrm{m})(\mathrm{K})^{-1}(\mathrm{~s})^{-3} ; \\
\rho_{0} & =8.954 \times 10^{3}(\mathrm{~kg})(\mathrm{m})^{-3} ; \\
\alpha_{t} & =17.8 \times 10^{-6}(\mathrm{~K})^{-} 1 ; \\
T_{0} & =293(\mathrm{~K}) ; \quad a=1 .
\end{aligned}
$$

The numerical computation had been carried out with the help of MATHEMATICA and MATLAB files for the length to mean radius ratio $\left(\eta=\frac{b-a}{R}\right)$, where $a=1, b>a, R=\frac{a+b}{2}$. Due to the presence of a dissipation term in the heat conduction equation, the frequency equation in the general complex transcendental equation provided us with a complex frequency value, $\omega^{m}=\omega_{R}^{m}+i \omega_{I}^{m}$, where $m$ was the mode number that corresponded to the roots of the transcendental Eq. (30) and $\omega_{R}^{m}$ and $\omega_{I}^{m}$ were the real part and imaginary parts of frequency $\omega^{m}$. The thermoelastic damping factor was given by:

$$
Q^{-1}=2\left|\frac{\omega_{I}^{m}}{\omega_{R}^{m}}\right| .
$$

The frequency shift due to thermal variations was defined as:

$$
\omega_{s}=\left|\frac{\omega_{R}^{m}-\omega_{o}^{m}}{\omega_{o}^{m}}\right| ;
$$

where $\omega_{o}^{m}$ was the frequency in elastic hollow cylinder.

The calculation of the roots of the dispersion relation Eq. (30) represented a major task and required a rather extensive effort of numerical computation. The frequency spectrum $\omega^{m}$ versus the different values of the length to mean radius ratio $\eta$ for the first twenty modes was computed by the interval halving method. The validation of the roots for the dispersion relation is presented in Table 1 .

The first twenty modes of non-dimensional frequency $\omega_{R}$ verses the length to radius ratio $\eta$ are presented in Fig. 1. It was observed that the non-dimensional frequency $\omega_{R}$ decreased with an increasing length to mean radius ratio. The thermoelastic damping $Q^{-1}$ versus $m$ for different values of the length to mean radius ratio $\eta$ are presented in Fig. 2, from which it is seen that the thermoelastic damping $Q^{-1}$ increased initially to attain its maximum peak value at the second mode before it decreased in order to become ultimately asymptotic with increasing $m$. Figure 3 shows the variation of the frequency shift $\omega_{s}$ versus $m$ for different values of the length to mean radius ratio $\eta$. It could be inferred that the frequency shift $\omega_{s}$ increased sharply to attain its maximum peak value at the second mode and then decreased to become ultimately asymptotic with increasing $m$.

\section{CONCLUSIONS}

The exact solution for a free vibration of thermoelastic hollow cylinder under GNIII model has been done with the help of the eigenvalue approach. The eigenvalue approach is applied successfully to get an explicit, totally analytic, and uniformly valid solution for the current problem. The validation of the roots for the dispersion relation is also presented. The closed form solution obtained here opens the scope of further studies in mathematics, science, and engineering disciplines.

\section{REFERENCES}

1 Lord, H. W. and Y. Shulman. A generalized dynamical theory of thermoelasticity, Journal of the Mechanics and Physics of Solids, 15(5),299-309, (1967).

2 Green, A. E. and K. A. Lindsay. Thermoelasticity, Journal of Elasticity, 2(1), 1-7 (1972).

3 Dhaliwal, R. S. and H. H. Sherief. Generalized thermoelasticity for anisotropic media, Quarterly of Applied Mathematics, 38(1), 1-8 (1980).

4 Sherief, H. H. and M. N. Anwar. A problem in generalized thermoelasticity for an infinitely long annular cylinder, International Journal of Engineering Science, 26(3), 301306, (1988).

5 Sherief, H. H. and M. A. Ezzat. A problem in generalized magneto-thermoelasticity for an infinitely long annular cylinder, Journal of Engineering Mathematics, 34(1-4), 387-402, (1998).

6 Abbas, I. A. and A. M. Zenkour. LS model on electromagneto-thermoelastic response of an infinite functionally graded cylinder, Composite Structures, 96, 89-96, (2013).

7 Abbas, I. A. Generalized magneto-thermoelastic interaction in a fiber-reinforced anisotropic hollow cylinder, International Journal of Thermophysics, 33(3): 567-579, (2012).

8 . Abd-alla, A. N. and I. Abbas. A problem of generalized magnetothermo-elasticity for an infinitely long, perfectly conducting cylinder. Journal of Thermal Stresses, 25(11), 1009-1025, (2002). 
9 Abbas, I. A. and H. M. Youssef. A nonlinear generalized thermoelasticity model of temperature-dependent materials using finite element method, International Journal of Thermophysics, 33(7), 1302-1313, (2012).

10 Green, A. E. and P. M. Naghdi. Thermoelasticity without energy dissipation, Journal of Elasticity, 31(3), 189-208, (1993).

11 Green, A. E. and P. M. Naghdi. On undamped heat waves in an elastic solid, Journal of Thermal Stresses, 15(2), 253264, (1992).

12 Abd El-Latief, A. M. and S. E. Khader. Exact solution of thermoelastic problem for a one-dimensional bar without energy dissipation, ISRN Mechanical Engineering, 2014, (2014).

13 Youssef, H. M. State-space approach to two-temperature generalized thermoelasticity without energy dissipation of medium subjected to moving heat source, Applied Mathematics and Mechanics (English Edition), 34(1),63-74, (2013).

14 Mukhopadhyay, S. and R. Kumar. A problem on thermoelastic interactions without energy dissipation in an unbounded medium with a spherical cavity, Proceedings of the National Academy of Sciences India Section A - Physical Sciences, 79(1), 135-140, (2009).

15 Sharma, S., K. Sharma, and R. R. Bhargava. Effect of viscosity on wave propagation in anisotropic thermoelastic with Green-Naghdi theory type-II and type-III, Materials Physics and Mechanics, 16(2), 144-158, (2013).

16 Prasad, R., S. Das, and S. Mukhopadhyay. A twodimensional problem of a mode i crack in a type III thermoelastic medium, Mathematics and Mechanics of Solids, 18(5), 506-523, (2013).

17 Othman, M. I. A. and S. Y. Atwa. Thermoelastic plane waves for an elastic solid half-space under hydrostatic initial stress of type III, Meccanica, 47(6), 1337-1347, (2012).

18 Abbas, I. A. A GN model for thermoelastic interaction in an unbounded fiber-reinforced anisotropic medium with a circular hole, Applied Mathematics Letters, 26(2), 232239, (2013).

19 Abbas, I. A. and A. M. Zenkour. The effect of rotation and initial stress on thermal shock problem for a fiber-reinforced anisotropic half-space using green-naghdi theory, Journal of Computational and Theoretical Nanoscience, 11(2), 331-338, (2014).

20 Hetnarski, R. B. and J. Ignaczak. Generalized thermoelasticity, Journal of Thermal Stresses, 22(4), 451-476, (1999).

21 Abbas, I. Natural frequencies of a poroelastic hollow cylinder, Acta Mechanica, 186(1-4), 229-237, (2006).

22 Abd-alla, A. N. and I. A. A. Abbas. Magnetoelastic longitudinal wave propagation in a transversely isotropic circular cylinder, Applied Mathematics and Computation, 127(23), 347-360, (2002).
23 Mykityuk, Y. I. Thermoelastic vibrations of a thick-walled cylinder of time-varying thickness, Soviet Applied Mechanics, 5(11), 1237-1240, (1972).

24 Zhitnyaya, V. G. The uncoupled problem of thermoelastic vibrations of a cylinder, Journal of Mathematical Sciences, 92(5), 4190-4192, (1998).

25 Marin, M. and M. Lupu. On harmonic vibrations in thermoelasticity of micropolar bodies, Journal of Vibration and Control, 4(5), 507-518, (1998).

26 Erbay, HA., S. Erbay, and S. Dost. Thermally induced vibrations in a generalized thermoelastic solid with a cavity, Journal of Thermal Stresses, 14(2), 161-171, (1991).

27 Sharma, J. N., H. Singh, and Y. D. Sharma. Vibration analysis of a transversely isotropic hollow cylinder by using the matrix Frobenius method, Journal of Thermal Stresses, 34(9), 934-957, (2011).

28 Nayfeh, A. H. and M. I. Younis. A model for thermoelastic damping in microplates, (2004)

29 Nayfeh, A. H. and M. I. Younis, Modeling and simulations of thermoelastic damping in microplates, Journal of $\mathrm{Mi}$ cromechanics and Microengineering, 14(12), 1711-1717, (2004).

30 Rezazadeh, G., et al. Thermoelastic damping in a microbeam resonator using modified couple stress theory, Acta Mechanica, 223(6), 1137-1152, (2012).

31 Das, N. C., A. Lahiri, and R. R. Giri. Eigenvalue approach to generalized thermoelasticity, Indian Journal of Pure and Applied Mathematics, 28(12), 1573-1594, (1997).

32 Abbas, I. A. Eigenvalue approach to fractional order generalized magneto-thermoelastic medium subjected to moving heat source, Journal of Magnetism and Magnetic Materials, 377, 452-459, (2015).

33 Abbas, I. A. Eigenvalue approach in a three-dimensional generalized thermoelastic interactions with temperaturedependent material properties, Computers \& Mathematics with Applications, 68(12), 2036-2056, (2014).

34 Abbas, I. A. Eigenvalue approach for an unbounded medium with a spherical cavity based upon twotemperature generalized thermoelastic theory, Journal of Mechanical Science and Technology, 28(10), 4193-4198, (2014).

35 Youssef, H. M. and I. A. Abbas. Fractional order generalized thermoelasticity with variable thermal conductivity, Journal of Vibroengineering, 16(8), 4077-4087, (2014).

36 Abbas, I. A. A GN model based upon two-temperature generalized thermoelastic theory in an unbounded medium with a spherical cavity, Applied Mathematics and Computation, 245, 108-115, (2014).

37 Abbas, I. Generalized magneto-thermoelasticity in a nonhomogeneous isotropic hollow cylinder using the finite element method, Archive of Applied Mechanics, 79(1), 41-50, (2009). 